\title{
Densely homogeneous fuzzy spaces
}

\author{
Samer Al Ghour \\ Department of Mathematics and Statistics, Jordan University of Science and Technology, Jordan
}

\begin{tabular}{l} 
Article Info \\
\hline Article history: \\
Received Jul 26, 2018 \\
Revised Apr 2, 2019 \\
Accepted Apr 11, 2019 \\
\hline
\end{tabular}

\begin{abstract}
We extend the concept of being densely homogeneous to include fuzzy topological spaces. We prove that our extension is a good extension in the sense of Lowen. We prove that a-cut topological space $\left(X, \mathfrak{J}_{\mathrm{a}}\right)$ of a DH fuzzy topological space $(X, \mathfrak{I})$ is DH in general only for $a=0$.
\end{abstract}

\section{Keywords:}

Cut topologies

Densely homogeneous

Fuzzy CDH

Good extension

\section{Corresponding Author:}

Samer Al Ghour,

Department of Mathematics and Statistics,

Jordan University of Science and Technology,

Irbid 22110, Jordan

Email: algore@just.edu.jo

\section{INTRODUCTION}

As defined in [1], the notion of a fuzzy set in a set $\mathrm{X}$ is a function from $\mathrm{X}$ into the closed interval [0,1]. Accordingly, Chang [2] introduced the notion of a fuzzy topological space on a non-empty set $\mathrm{X}$ as a collection of fuzzy sets on $\mathrm{X}$, closed under arbitrary suprema and finite infima and containing the constant fuzzy sets 0 and 1 . Mathematicians extended many topological concepts to include fuzzy topological spaces such as: separation axioms, connectedness, compactness and metrizability. Several fuzzy homogeneity concepts were discussed in [3-11]. A separable topological space $(X, \tau)$ is countable dense homogeneous $(\mathrm{CDH})$ [12] if given any two countable dense subsets $A$ and $B$ of $(X, \tau)$ there is a homeomorphism $\mathrm{f:}(\mathrm{X}, \tau) \rightarrow(\mathrm{X}, \tau)$ such that $\mathrm{f}(\mathrm{A})=\mathrm{B}$.

The study of CDH topological spaces and their related concepts is still a hot area of research, as appears in [13-20] and other papers. Recently, authors in [9] extended CDH topological property to include fuzzy topological spaces. They proved that their extension is a good extension in the sense of Lowen, and proved that a-cut topological space $\left(\mathrm{X}, \mathfrak{J}_{\mathrm{a}}\right)$ of a CDH fuzzy topological space $(\mathrm{X}, \mathfrak{J})$ is $\mathrm{CDH}$ in general only for $\mathrm{a}=0$. For the purpose of dealing with non-separable topological spaces, authors in [21] modified the definition of $\mathrm{CDH}$ topological spaces as follows: A subset $\mathrm{A}$ of a topological space $(\mathrm{X}, \tau)$ is called a $\sigma$-discrete set if it is the union of countably many sets, each with the relative topology, being a discrete topological space. A topological space $(X, \tau)$ is densely homogeneous $(\mathrm{DH})$ provided $(\mathrm{X}, \tau)$ has a $\sigma$-discrete subset which is dense in $(X, \tau)$ and if $A$ and $B$ are two such $\sigma$-discrete subsets of $(X, \tau)$ there is a homeomorphism $\mathrm{f}:(\mathrm{X}, \tau) \rightarrow(\mathrm{X}, \tau)$ such that $\mathrm{f}(\mathrm{A})=\mathrm{B}$. It is known that $\mathrm{CDH}$ and $\mathrm{DH}$ topological concepts are independent. The study of DH topological spaces is continued in [22-28] and other papers. As a main goal of the present work we will show how the definition of DH topological spaces can be modified in order to define a good extension of it in fuzzy topological spaces. We will give relationships between CDH and DH fuzzy. 
Throughout this paper, if $X$ is a set, then $|X|=\operatorname{Card} X$ will denote its cardinality. We write $\mathbb{Q}(\operatorname{resp} . \mathbb{N})$ to denote the set of all rational numbers (resp. natural numbers). The closure of a fuzzy set $\lambda$ in a fuzzy topological space $(X, \mathfrak{I})$ will be denoted by $\operatorname{Cl}(\lambda)$. Associated with a given topological space $(X, \tau)$ and arbitrary subset $A$ of $X$, we denote the relative topology on $A$ by $\tau_{A}$, the closure of $A$ by $\operatorname{Cl}(A)$ and the boundary of $A$ by $B d(A)$.topological spaces as well as we will deal with cut topological spaces.

\section{PRELIMINARIES}

In this paper we shall follow the notations and definitions of [29] and [30]. If $(X, \tau)$ is a topological space, then the class of all lower semi-continuous functions from $(X, \tau)$ to $\left([0,1], \tau_{u}\right)$, where $\tau_{u}$ is the usual Euclidean topology on $[0,1]$, is a fuzzy topology on $X$. This fuzzy topology is denoted by $\omega(\tau)$. The following definitions and propositions will be used in the sequel:

Definition 2.1. [9] Let $X$ be a non-empty set, $A$ be a non-empty subset of $X$ and $P$ be a collection of fuzzy points in $X$. Then

- $\mathbb{Q}(A)$ will denote the set

$$
\mathbb{Q}(A)=\left\{x_{r}: x_{r} \text { is a fuzzy point with } x \in A \text { and } \mathrm{r} \in \mathbb{Q} \cap(0,1)\right\} \text {. }
$$

- $\quad$ The support of $P$, denoted by $S(P)$, is defined by

$S(P)=\left\{x: x_{a} \in P\right.$ for some $\left.a\right\}$.

Definition 2.2. [21] A subset $A$ of a topological space $(X, \tau)$ is called a $\sigma$-discrete set if it is the union of countably many sets, each with the relative topology, being a discrete topological space. Definition 2.3. [21] A topological space $(X, \tau)$ is called densely homogeneous (DH) iff

- $\quad X$ has a $\sigma$-discrete dense subset.

- If $A$ and $B$ are two $\sigma$-discrete dense subsets of $X$, then there is a homeomorphism $h:(X, \tau) \rightarrow(X, \tau)$ such that $h(A)=B$.

Definition 2.4. [31] Associated with a given fuzzy topological space (X, $\mathfrak{I})$ and arbitrary subset $M$ of $X$, we define the induced fuzzy topology on $M$ or the relative topology on $M$ by

$$
\mathfrak{I}_{M}=\{\lambda \mid M: \lambda \in \mathfrak{I}\}
$$

Definition 2.5. [9] A fuzzy topological space $(X, \mathfrak{I})$ is said to be semi-discrete iff for any $\mathrm{x} \in \mathrm{X}$, there exists a fuzzy point or a fuzzy crisp point $x_{a}$ for some a with $x_{a} \in \mathfrak{I}$. Definition 2.6. [32] Let (X, I) be a fuzzy topological space and let $P$ be a collection of fuzzy points of $X$. Then $P$ is said to be

- Dense(I) if for every non-zero fuzzy open set $\lambda$ there exists $p \in P$ such that $p \in \lambda$.

- Dense(II) if $\operatorname{Cl}\left(\mathrm{U}_{p \in P} p\right)=1$.

Definition 2.7. [9] A fuzzy topological space $(X, \mathfrak{I})$ is called separable iff there exists a countable dense(I) collection of fuzzy points of $X$. Definition 2.8. [33] A property $\mathrm{P}_{f}$ of a fuzzy topological space is said to be a good extension of the property $\mathrm{P}$ in classical topology iff whenever the fuzzy topological space is topologically generated, say by $(X, \tau)$, then $(X, \omega(\tau))$ has property $\mathrm{P}_{f}$ iff $(X, \tau)$ has property $\mathrm{P}$.

Definition 2.9. [34] Let $(X, \mathfrak{I})$ be a fuzzy topological space and $a \in[0,1)$. The topology $\left\{\lambda^{-1}(a, 1]: \lambda \in \mathfrak{I}\right\}$ on $\mathrm{X}$ is called $a$-cut topological space of $(X, \mathfrak{J})$ and will be denoted by $\mathfrak{J}_{a}$. The topological space $\left(X, \mathfrak{I}_{a}\right)$ will be called $a$-cut topological space of $(X, \mathfrak{I})$. Definition 2.10. [9] A fuzzy topological space $(X, \mathfrak{I})$ is said to be countable dense homogeneous; denoted $\mathrm{CDH}$; iff

- $(X, \mathfrak{J})$ is separable.

- If $P$ and $W$ are two countable dense(I) collections of fuzzy points of $X$, then there is a fuzzy homeomorphism $h:(X, \mathfrak{I}) \rightarrow(X, \mathfrak{I})$ such that $h(S(P))=S(W)$.

Proposition 2.11. [9] Let $(\mathrm{X}, \mathfrak{I})$ be a fuzzy topological space and let $P$ be a collection of fuzzy points of $\mathrm{X}$. Then we have the following

- If $P$ is dense (I), then $\mathbb{Q}(\mathrm{S}(P))$ is dense(II).

- If $P$ is dense (II), then $\mathbb{Q}(\mathrm{S}(P))$ is dense(I).

Proposition 2.12. [9] Let $(X, \tau)$ be a topological space, $A \subseteq X$, and $P$ be a collection of fuzzy points of $X$. Then we have the following

- If $A$ is dense in $(X, \tau)$, then $\mathbb{Q}(A)$ is dense $(\mathrm{I})$ in $(X, \omega(\tau))$.

- If $P$ is dense $(\mathrm{I})$ in $(X, \omega(\tau))$, then $S(P)$ is dense in $(X, \tau)$.

Proposition 2.13. [35] Let $\left(X, \tau_{1}\right)$ and $\left(Y, \tau_{2}\right)$ be two topological spaces. Then $f:\left(X, \tau_{1}\right) \rightarrow\left(Y, \tau_{2}\right)$ is continuous iff $f:\left(X, \omega\left(\tau_{1}\right)\right) \rightarrow\left(Y, \omega\left(\tau_{2}\right)\right)$ is fuzzy continuous. Proposition 2.14. [9] Let $f:\left(X, \mathfrak{I}_{1}\right) \rightarrow\left(Y, \mathfrak{I}_{2}\right)$ be a fuzzy homeomorphism map and $P$ be a collection of fuzzy points of $X$. Then we have the following 
$-\quad S(f(P))=f(S(P))$.

- $\quad$ If $P$ is dense $(\mathrm{I})$ in $\left(X, \mathfrak{J}_{1}\right)$, then $f(P)$ is dense(I) in $\left(Y, \mathfrak{J}_{2}\right)$.

following

Proposition 2.15. [9] Let $(X, \mathfrak{I})$ be a semi-discrete fuzzy topological space. Then we have the

- $\quad$ If $P$ is countable dense $(\mathrm{I})$ in $(X, \mathfrak{I})$, then $S(P)=X$.

- $\quad(X, \mathfrak{I})$ is separable iff $X$ is countable.

Proposition 2.16. [9] Let $X$ be a countable set and let $(X, \mathfrak{I})$ be a fuzzy topological space.

Then $(X, \mathfrak{I})$ is CDH iff $(X, \mathfrak{I})$ is a semi-discrete fuzzy topological space. Proposition 2.17. [34] Let $(\mathrm{X}, \mathfrak{I})$ be a fuzzy topological space and let $f:(X, \mathfrak{I}) \rightarrow(X, \mathfrak{J})$ be a fuzzy continuous (homeomorphism) map. Then $f:\left(X, \mathfrak{J}_{a}\right) \rightarrow\left(X, \mathfrak{J}_{a}\right)$ is continuous (homeomorphism) for all $a \in[0,1)$. Proposition 2.18. [9] Let $(X, \mathfrak{I})$ be a fuzzy topological space. Let $A$ be a subset of $X$ and let $P$ be a collection of fuzzy points of $X$. Then we have the following

- If $A$ is dense in $\left(X, \mathfrak{I}_{0}\right)$, then $\mathbb{Q}(A)$ is dense $(\mathrm{I})$ in $(X, \mathfrak{I})$.

- $\quad$ If $P$ is dense $(\mathrm{I})$ in $(X, \mathfrak{I})$, then $S(P)$ is dense in $\left(X, \mathfrak{J}_{0}\right)$.

\section{DH FUZZY TOPOLOGICAL SPACES}

In this section, we will define DH fuzzy topological spaces. We will prove that our new concept is a fuzzy topological property and a good extension of DH topological property in the sense of Lowen. Definition 3.1. A collection $P$ of fuzzy points of a fuzzy topological space $(X, \mathfrak{I})$ is said to be

- $\sigma$-semi-discrete iff $S(P)=\bigcup_{n=1}^{\infty} A_{n}$ with $\left(A_{n}, \widetilde{J}_{A_{n}}\right)$ is semi-discrete for all $n \in \mathbb{N}$.

- $\sigma$-semi-discrete dense (I) iff $P$ is $\sigma$-semi-discrete and $P$ is dense (I).

- $\sigma$-semi-discrete dense (II) iff $P$ is $\sigma$-semi-discrete and $P$ is dense (II).

Definition 3.2. A fuzzy topological space $(X, \mathfrak{I})$ is said to be densely homogeneous (DH) iff

- $\quad(X, \mathfrak{I})$ has a $\sigma$-semi-discrete dense(I) collection of fuzzy points.

- If $P$ and $W$ are two $\sigma$-semi-discrete dense (I) collections of fuzzy points of $(X, \mathfrak{I})$, then there is a fuzzy homeomorphism $h:(X, \mathfrak{I}) \rightarrow(X, \mathfrak{I})$ such that $h(S(P))=S(W)$.

Lemma 3.3. Let $(X, \mathfrak{I})$ be a fuzzy topological space and $P$ be a $\sigma$-semi-discrete collection of fuzzy points of $X$. Then $\mathbb{Q}(S(P))$ is a $\sigma$-semi-discrete collection of fuzzy points of $(X, \mathfrak{J})$. Proof. It is easy to see that $S(P)=S(\mathbb{Q}(S(P))$ ) and hence the result is obvious. Theorem 3.4. A fuzzy topological space $(\mathrm{X}, \mathfrak{I})$ is DH iff

- $\quad(X, \mathfrak{I})$ has a $\sigma$-semi-discrete dense(II) collection of fuzzy points.

- If $P$ and $W$ are two $\sigma$-semi-discrete dense (II) collections of fuzzy points of $(X, \mathfrak{J})$, then there is a fuzzy homeomorphism $h:(X, \mathfrak{I}) \rightarrow(X, \mathfrak{I})$ such that $h(S(P))=S(W)$.

Proof. If $(X, \mathfrak{I})$ is $\mathrm{DH}$, then $(X, \mathfrak{I})$ has a $\sigma$-semi-discrete dense (I) collection of fuzzy points $P$. By Proposition 2.11 (i), $\mathbb{Q}(S(P))$ is dense (II) and by Lemma 3.3, $\mathbb{Q}(S(P))$ is $\sigma$-semi-discrete. Let $P$ and $W$ be any two $\sigma$-semi-discrete dense (II) collections of fuzzy points of $(X, \mathfrak{J})$. Then by Proposition 2.11 (ii) and Lemma 3.3, $\mathbb{Q}(S(P))$ and $\mathbb{Q}(S(W))$ are both $\sigma$-semi-discrete dense (I) collections of fuzzy points of $(X, \mathfrak{J})$. Then there is a fuzzy homeomorphism $h:(X, \mathfrak{I}) \rightarrow(X, \mathfrak{J})$ such that $h(S(\mathbb{Q}(S(P))))=S(\mathbb{Q}(S(W)))$. Thus, $h(S(P))=S(W)$. The proof of the other direction of this theorem is similar to the above one. Lemma 3.5. Let $(X, \tau)$ be a topological space. Let $A$ be a non-empty subset of $X$ and $P$ be a collection of fuzzy points of $X$. Then

- $\tau_{A}$ is the discrete topology iff $\left(A, \omega(\tau)_{A}\right)$ is semi-discrete.

- If $A$ is $\sigma$-discrete in $(X, \tau)$, then $\mathbb{Q}(A)$ is $\sigma$-semi-discrete in $(X, \omega(\tau))$.

- If $P$ is $\sigma$-semi-discrete in $(X, \omega(\tau))$, then $S(P)$ is $\sigma$-discrete in $(X, \tau)$.

Proof. (i) Suppose that $\tau_{A}$ is the discrete topology and let $x \in A$. Then there exists $U \in \tau$ such that $\{x\}=U \cap A$. So, $X_{U} \cap X_{A}=X_{U \cap A}=X_{\{x\}} \in \omega(\tau)_{A}$. But clearly $\omega(\tau)_{A}$ is the crisp point with support $x$. Conversely, suppose that $\left(A, \omega(\tau)_{A}\right)$ is a semi-discrete fuzzy topological space and let $x \in A$. Then there exists a fuzzy point or a fuzzy crisp point $x_{a}$ such that $x_{a} \in \omega(\tau)_{A}$. Choose $\lambda \in \omega(\tau)$ such that $x_{a}=\lambda \cap x_{A}$. Thus, $\{x\}=\lambda^{-1}(0,1] \cap A$ and hence $\{x\} \in \tau_{A}$.

(ii) Since $A$ is $\sigma$-discrete in $(X, \tau)$, then $A=\bigcup_{n=1}^{\infty} A_{n}$ with $\tau_{A_{n}}$ is the discrete topology for all $n \in \mathbb{N}$. So, by part (i) $\left(A_{n}, \omega(\tau)_{A_{n}}\right)$ is semi-discrete for all $n$. Since $S(\mathbb{Q}(A))=A$, then $\mathbb{Q}(A)$ is $\sigma$-semi-discrete in $(X, \omega(\tau))$. (iii) Since $P$ is $\sigma$-semi-discrete in $(X, \omega(\tau))$, then $S(P)=\bigcup_{n=1}^{\infty} A_{n}$ with $\left(A_{n}, \omega(\tau)_{A_{n}}\right)$ is semidiscrete for all $n \in \mathbb{N}$. So, by part (i) $\tau_{A_{n}}$ is the discrete topology for all $n$. Therefore, $S(P)$ is $\sigma$-discrete in $(X, \tau)$.

Theorem 3.6. Let $(X, \tau)$ be a topological space. Then $(X, \tau)$ is $\mathrm{DH}$ iff $(X, \omega(\tau))$ is $\mathrm{DH}$. Proof. Suppose that $(X, \tau)$ is DH. Then $(X, \tau)$ has a $\sigma$-discrete dense subset $A$. By Lemma 3.5 (ii) and 
Proposition 2.12 (i), $\mathbb{Q}(A)$ is $\sigma$-semi-discrete dense (I) in $(X, \omega(\tau))$. Let $P$ and $W$ be two $\sigma$-semi-discrete dense(I) collections of fuzzy points of $(X, \omega(\tau))$. Then by Lemma 3.5 (iii) and Proposition 2.12 (ii), $S(P)$ and $S(W)$ are both $\sigma$-discrete dense subsets of $(X, \tau)$. Thus, there is a homeomorphism $h:(X, \tau) \rightarrow(X, \tau)$ such that $h(S(P))=S(W)$. Proposition 2.13 ends the proof of this direction.

Conversely if $(X, \omega(\tau))$ is $\mathrm{DH}$, then $(X, \omega(\tau))$ has a $\sigma$-discrete dense (I) collection of fuzzy points $P$. By Lemma 3.5 (iii) and Proposition 2.12 (ii), $S(P)$ is $\sigma$-discrete dense in $(X, \tau)$. Let $A$ and $B$ be two $\sigma$-discrete dense subsets of $(X, \tau)$. Then by Lemma 3.5 (ii) and Proposition 2.12 (i), $\mathbb{Q}(A)$ and $\mathbb{Q}(B)$ are both $\sigma$-semi-discrete dense (I) collections of fuzzy points of $(X, \omega(\tau))$. Thus, there is a fuzzy homeomorphism $h:(X, \omega(\tau)) \rightarrow(X, \omega(\tau))$ such that $h(S(\mathbb{Q}(A)))=S(\mathbb{Q}(A))$. So, $h(A)=B$. Proposition 2.13 ends the proof of this direction.

Corollary 3.7. DH in fuzzy topological spaces is a good extension of DH in topological spaces. Recall that a property $\mathrm{P}_{f}$ of fuzzy topological spaces is called a fuzzy topological property if whenever $\left(X, \mathfrak{I}_{1}\right)$ possesses $\mathrm{P}_{f}$ and $h:\left(X, \mathfrak{I}_{1}\right) \rightarrow\left(Y, \mathfrak{I}_{2}\right)$ is a fuzzy homeomorphism, then $\left(Y, \mathfrak{I}_{2}\right)$ possesses $\mathrm{P}_{f}$. Lemma 3.8. Let $f: X \rightarrow Y$ be a bijective map. Then

- For any two fuzzy sets $\lambda, \mu$ in $X, f(\lambda \cap \mu)=f(\lambda) \cap f(\mu)$.

- For any $A \subseteq X, f\left(\chi_{A}\right)=\chi_{f(A)}$.

Proof. Straightforward. Lemma 3.9. Let $f:\left(X, \mathfrak{I}_{1}\right) \rightarrow\left(Y, \mathfrak{I}_{2}\right)$ be a fuzzy homeomorphism. Let $A$ be a non-empty subset of $X$ and $P$ be a collection of fuzzy points of $X$. Then

- If $\left(A,\left(\mathfrak{I}_{1}\right)_{A}\right)$ is semi-discrete, then $\left(f(A),\left(\mathfrak{I}_{2}\right)_{f(A)}\right)$ is semi-discrete.

- $\quad$ If $P$ is $\sigma$-semi-discrete, then $f(P)$ is $\sigma$-semi-discrete.

Proof. (i) Let $y \in f(A)$, say $y=f(x)$ for some $x \in A$. Since $\left(A,\left(\mathfrak{I}_{1}\right)_{A}\right)$ is semi-discrete, there exists $r \in(0,1]$ such that $x_{r} \in\left(\mathfrak{I}_{1}\right)_{A}$. Choose $\lambda \in \mathfrak{I}_{1}$ such that $x_{r}=\lambda \cap \mathcal{X}_{A}$. Then by Lemma $3.8 y_{r}=(f(x))_{r}=f\left(x_{r}\right)=f\left(\lambda \cap X_{A}\right)=f(\lambda) \cap f\left(X_{A}\right)=f(\lambda) \cap X_{f(A)}$. Since $f$ is fuzzy open, it follows that $y_{r} \in\left(\mathfrak{I}_{2}\right)_{f(A)}$. ii) Since $P$ is $\sigma$-semi-discrete, $S(P)=\bigcup_{n=1}^{\infty} A_{n}$ with $\left(A_{n}, \mathfrak{I}_{A_{n}}\right)$ is semi-discrete for all $n \in \mathbb{N}$. By Proposition 2.14 (i), $S(f(P))=f(S(P))=f\left(\bigcup_{n=1}^{\infty} A_{n}\right)=\bigcup_{n=1}^{\infty} f\left(A_{n}\right)$. Also, by (i) we have $\left(f\left(A_{n}\right),\left(\mathfrak{I}_{2}\right)_{f\left(A_{n}\right)}\right)$ is semi-discrete for all $n \in \mathbb{N}$. It follows that $f(P)$ is $\sigma$-semi-discrete.

Theorem 3.10. In fuzzy topological spaces, "Being "DH" is a fuzzy topological property. Proof. Assume $\left(X, \mathfrak{I}_{1}\right)$ is a DH fuzzy topological space and let $f:\left(X, \mathfrak{I}_{1}\right) \rightarrow\left(Y, \mathfrak{I}_{2}\right)$ be a fuzzy homeomorphism where $\left(Y, \mathfrak{I}_{2}\right)$ is a fuzzy topological space. Choose a $\sigma$-semi-discrete dense(I) collection of fuzzy points $P$ of $\left(X, \mathfrak{J}_{1}\right)$. According to Lemma 3.9 (ii) and Proposition 2.14 (ii), $f(P)$ will be $\sigma$-semi-discrete dense(I) in $\left(Y, \mathfrak{J}_{2}\right)$. Let $P$ and $W$ be any two $\sigma$-semi-discrete dense(I) collections of fuzzy points of $\left(Y, \mathfrak{J}_{2}\right)$. Then by Lemma 3.9 (ii) and Proposition 2.14 (ii), $f^{-1}(P)$ and $f^{-1}(W)$ are two $\sigma$-semi-discrete dense(I) collections of fuzzy points of $\left(X, \mathfrak{J}_{1}\right)$. Since $\left(X, \mathfrak{J}_{1}\right)$ is DH, there is a fuzzy homeomorphism $h:\left(X, \mathfrak{J}_{1}\right) \rightarrow\left(X, \mathfrak{J}_{1}\right)$ such that $h\left(S\left(f^{-1}(P)\right)\right)=S\left(f^{-1}(W)\right)$. Define $g:\left(Y, \mathfrak{J}_{2}\right) \rightarrow\left(Y, \mathfrak{I}_{2}\right)$ by $g=f \circ h \circ f^{-1}$. Then $g$ is a fuzzy homeomorphism. Using Proposition 2.14 (i), we can see that $g(S(P))=S(W)$.

\section{RELATIONSHIPS BETWEEN DH AND CDH FUZZY TOPOLOGICAL SPACES}

In this section, we will give some relationships between $\mathrm{DH}$ and $\mathrm{CDH}$ fuzzy topological spaces.

The following useful lemma follows easily: Lemma 4.1. Let $(X, \mathfrak{I})$ be a fuzzy topological space and P be a collection of fuzzy points of $X$ with $S(P)$ is countable and non-empty. Then $P$ is $\sigma$-semi-discrete. Theorem 4.2. Let $(X, \mathfrak{J})$ be a fuzzy topological space for which $X$ is countable. Then $(X, \mathfrak{I})$ is DH iff $(X, \mathfrak{I})$ is semi-discrete. Proof. Since the result is obvious when $|X|=1$, we will assume that $|X|>1$. Suppose that $(X, \mathfrak{I})$ is $\mathrm{DH}$ and assume on the contrary that $(\mathrm{X}, \mathfrak{J})$ is not semi-discrete. Then there exists $\mathrm{y} \in \mathrm{X}$ such that $\mathrm{y}_{\mathrm{a}} \notin \mathfrak{I}$ for all $0<\mathrm{a} \leq 1$. Set $\mathrm{P}=\mathbb{Q}(\mathrm{X})$ and $\mathrm{W}=\mathbb{Q}(\mathrm{X} \backslash\{\mathrm{y}\})$. It is not difficult to see that $\mathrm{P}$ and $\mathrm{W}$ are dense (I). Also, by Lemma 4.1, P and W are $\sigma$-semi-discrete. So there is a fuzzy homeomorphism $h:(X, \mathfrak{I}) \rightarrow(X, \mathfrak{I})$ such that $h(S(P))=S(W)$, therefore, $h(X)=X \backslash\{y\}$ which is a contradiction since $h$ is an onto map.

Conversely, suppose that $(X, \mathfrak{I})$ is semi-discrete. Then by Proposition 2.15 (ii), $(X, \mathfrak{I})$ is separable. Choose a countable dense (I) collection of fuzzy points $\mathrm{P}$. Then $\mathrm{S}(\mathrm{P})$ is countable and by Lemma $4.1, \mathrm{P}$ is $\sigma$-semi-discrete. Let $\mathrm{P}$ and $\mathrm{W}$ be any two $\sigma$-semi-discrete dense(I) collections of fuzzy points. Then by Proposition 2.15 (i), S(P) $=\mathrm{S}(\mathrm{W})=\mathrm{X}$ and the identity fuzzy map completes the proof. Corollary 4.3. Let $(X, \mathfrak{J})$ be a fuzzy topological space for which $X$ is countable. Then $(X, \mathfrak{I})$ is CDH iff $(X, \mathfrak{J})$ is DH. Proof. Follows from Proposition 2.16 and Theorem 4.2. Theorem 4.4. If (X, J) is separable and DH fuzzy topological space, then $(\mathrm{X}, \mathfrak{I})$ is $\mathrm{CDH}$. Proof. Follows from the definitions and Lemma 4.1 .

Recall that a fuzzy topological space $(X, \mathfrak{I})$ is hereditarily separable if every subspace of $(X, \mathfrak{I})$ is separable. Recall that a fuzzy topological space is second countable if it has a countable base. It is well known that second countable fuzzy topological spaces are hereditarily separable. Lemma 4.5 . If (X, I) is a hereditarily separable fuzzy topological space and $\mathrm{P}$ is a $\sigma$-semi-discrete collection of fuzzy points of $(X, \mathfrak{J})$, 
then $S(P)$ is countable. Proof. Since $P$ is $\sigma$-semi-discrete, then $S(P)=\bigcup_{n=1}^{\infty} A_{n}$ with $\left(A_{n}, \mathfrak{J}_{A_{n}}\right)$ is semidiscrete for all $n \in \mathbb{N}$. Since $(X, \mathfrak{J})$ is hereditarily separable, then for each $n \in \mathbb{N},\left(A_{n}, \mathfrak{J}_{A_{n}}\right)$ is separable and by Proposition 2.15 (ii) it follows that $A_{n}$ is countable. Thus, $S(P)$ is countable.

Theorem 4.6. If $(X, \mathfrak{I})$ is hereditarily separable and $\mathrm{CDH}$ fuzzy topological space, then $(\mathrm{X}, \mathfrak{I})$ is $\mathrm{DH}$. Proof. Since $(X, \mathfrak{I})$ is hereditarily separable, then it is separable. So, there exists a countable dense (I) collection of fuzzy points $\mathrm{P}$ and by Lemma $4.1, \mathrm{P}$ is $\sigma$-semi-discrete. Let $\mathrm{P}$ and $\mathrm{W}$ be two $\sigma$-semi-discrete dense(I) collections of fuzzy points. Then by Lemma 4.5, S(P) and S(W) are countable. By Proposition 2.11, $\mathbb{Q}(\mathrm{S}(\mathrm{P}))$ and $\mathbb{Q}(\mathrm{S}(\mathrm{W}))$ are countable dense(I). Since $(\mathrm{X}, \mathfrak{J})$ is $\mathrm{CDH}$, there is a fuzzy homeomorphism $\mathrm{h}:(\mathrm{X}, \mathfrak{I}) \rightarrow(\mathrm{X}, \mathfrak{I})$ such that $\mathrm{h}(\mathrm{S}(\mathrm{P}))=\mathrm{h}(\mathrm{S}(\mathbb{Q}(\mathrm{S}(\mathrm{P}))))=\mathrm{S}(\mathbb{Q}(\mathrm{S}(\mathrm{W})))=\mathrm{S}(\mathrm{W})$. Corollary 4.7. Let $(\mathrm{X}, \mathfrak{I})$ be a hereditarily separable fuzzy topological space. Then $(X, \mathfrak{I})$ is CDH iff $(X, \mathfrak{I})$ is DH. Proof. Follows from Theorems 4.4 and 4.6. Corollary 4.8. Let $(X, \mathfrak{I})$ be a second countable fuzzy topological space. Then $(X, \mathfrak{I})$ is $\mathrm{CDH}$ iff $(\mathrm{X}, \mathfrak{J})$ is $\mathrm{DH}$.

\section{CUT TOPOLOGICAL SPACES}

In this section we will mainly show that a-cut topological space $\left(X, \mathfrak{I}_{\mathrm{a}}\right)$ of a fuzzy topological $(\mathrm{X}, \mathfrak{I})$ is $\mathrm{DH}$ in general only if $\mathrm{a}=0$. Lemma 5.1 . Let $(\mathrm{X}, \mathfrak{I})$ be a fuzzy topological space. Let B be non-empty subset of $\mathrm{X}$ and let $\mathrm{P}$ be a collection of fuzzy points of $\mathrm{X}$. Then

- $\left(B, \mathfrak{I}_{B}\right)$ is semi-discrete iff $\left(\mathfrak{I}_{0}\right)_{B}$ is the discrete topology on $B$.

- If $B$ is $\sigma$-discrete in $\left(X, \mathfrak{J}_{0}\right)$, then $\mathbb{Q}(B)$ is $\sigma$-semi-discrete in $(X, \mathfrak{J})$.

- If $P$ is $\sigma$-semi-discrete in $(X, \mathfrak{I})$, then $S(P)$ is $\sigma$-discrete in $\left(X, \mathfrak{I}_{0}\right)$.

Proof. (i) Suppose that $\left(B, \mathfrak{J}_{B}\right)$ is semi-discrete and let $x \in B$. Then there exists a fuzzy point or a fuzzy crisp point $\mathrm{x}_{\mathrm{a}}$ for some a with $\mathrm{x}_{\mathrm{a}} \in \mathfrak{I}_{\mathrm{B}}$. Choose $\lambda \in \mathfrak{I}$ such that $\mathrm{x}_{\mathrm{a}}=\lambda \cap \mathcal{X}_{\mathrm{B}}$. Then $\left(\lambda \cap \mathcal{X}_{\mathrm{B}}\right)(\mathrm{x})=$ $\min \left\{\lambda(\mathrm{x}), \mathcal{X}_{\mathrm{B}}(\mathrm{x})\right\}>0$ and so, $\{\mathrm{x}\}=\lambda^{-1}(0,1] \cap \mathrm{B} \in\left(\mathfrak{I}_{0}\right)_{\mathrm{B}}$. Conversely, suppose that $\left(\mathfrak{I}_{0}\right)_{\mathrm{B}}$ is the discrete topology on $\mathrm{B}$ and let $\mathrm{x} \in \mathrm{B}$. Then there exists $\lambda \in \mathfrak{I}$ such that $\{\mathrm{x}\}=\lambda^{-1}(0,1] \cap \mathrm{B}$. Now, $\lambda \cap \mathcal{X}_{\mathrm{B}}$ is the fuzzy or crisp point $\mathrm{x}_{\lambda(\mathrm{x})}$, on the other hand, $\lambda \cap \mathcal{X}_{\mathrm{B}} \in \mathfrak{J}_{\mathrm{B} \text {. }}$ ii) Since $\mathrm{B}$ is $\sigma$-discrete in $\left(\mathrm{X}, \mathfrak{J}_{0}\right)$, then $\cup_{\mathrm{n}=1}^{\infty} \mathrm{B}_{\mathrm{n}}$ with $\left(\mathfrak{J}_{0}\right)_{\mathrm{B}_{n}}$ is the discrete topology for all $\mathrm{n} \in \mathbb{N}$. By (i), $\mathfrak{J}_{\mathrm{B}_{\mathrm{n}}}$ is semi-discrete for all $\mathrm{n} \in \mathbb{N}$. Since $\mathrm{S}(\mathbb{Q}(\mathrm{B}))=\mathrm{B}$, then $\mathbb{Q}(B)$ is $\sigma$-discrete in $(X, \mathfrak{J})$. iii) Since $P$ is $\sigma$-discrete in $(X, \mathfrak{J})$, then $S(P)=\cup_{n=1}^{\infty} A_{n}$ with $\mathfrak{I}_{A_{n}}$ is semidiscrete for all $n \in \mathbb{N}$. By (i), $\left(\mathfrak{I}_{0}\right)_{A_{n}}$ is the discrete topology on $A_{n}$ for all $n \in \mathbb{N}$. It follows that $S(P)$ is $\sigma$ discrete in $\left(X, \mathfrak{J}_{0}\right)$. Theorem 5.2. If $(X, \mathfrak{I})$ is a DH fuzzy topological space, then $\left(\mathrm{X}, \mathfrak{I}_{0}\right)$ is $\mathrm{DH}$.

Proof. Suppose that $(X, \mathfrak{I})$ is DH. Then $(X, \mathfrak{I})$ has a $\sigma$-semi-discrete dense(I) collection of fuzzy points P. By Lemma 5.1 (iii) and Proposition 2.18 (ii), S(P) is $\sigma$-discrete dense in (X, $\left.\mathfrak{J}_{0}\right)$. Let A and B be any two $\sigma$-discrete dense sets in $\left(\mathrm{X}, \mathfrak{I}_{0}\right)$. Then by Lemma 5.1 (ii) and Proposition 2.18 (i), $\mathbb{Q}(\mathrm{A})$ and $\mathbb{Q}(\mathrm{B})$ are $\sigma$-semi-discrete dense $(I)$ in $(X, \mathfrak{I})$. Since $(X, \mathfrak{I})$ is $D H$, there exists a fuzzy homeomorphism $\mathrm{h}:(\mathrm{X}, \mathfrak{I}) \rightarrow(\mathrm{X}, \mathfrak{I})$ such that $\mathrm{h}(\mathrm{S}(\mathbb{Q}(\mathrm{A})))=\mathrm{S}(\mathbb{Q}((\mathrm{B})))$. By Proposition 2.17 , h: $\left(\mathrm{X}, \mathfrak{J}_{0}\right) \rightarrow\left(\mathrm{X}, \mathfrak{I}_{0}\right)$ is a homeomorphism. On the other hand, $S(\mathbb{Q}(A))=A$ and $S(\mathbb{Q}(B))=B$. Therefore, $\left(X, \mathfrak{J}_{0}\right)$ is $D H$. The following proposition is well known: equivalent:

Proposition 5.3. Let $(X, \tau)$ be a topological space with $\mathrm{X}$ is countable. Then the following are

- $(X, \tau)$ is $\mathrm{CDH}$.

- $\tau$ is the discrete topology on $X$.

$-(X, \tau)$ is DH.

Theorem 5.4. Let $X$ be a countable set and let $(X, \mathfrak{I})$ be a fuzzy topological space. Then the following are equivalent:

$-(X, \mathfrak{J})$ is $\mathrm{DH}$.

$-(X, \mathfrak{J})$ is $\mathrm{CDH}$.

- $\left(X, \mathfrak{I}_{0}\right)$ is DH.

$-\left(X, \mathfrak{I}_{0}\right)$ is $\mathrm{CDH}$.

Proof. Follows from Theorem 4.2, Lemma 5.1 (i) and Proposition 5.3. In fact if a $>0$, then (X, I) being DH does not imply, in general, that $\left(X, \mathfrak{J}_{\mathrm{a}}\right)$ is DH. This will be explained in the following counterexample: Example 5.5. For fixed $0<\mathrm{a}<1$, let $\mathrm{X}=\{\mathrm{x}, \mathrm{y}\}$ and define $\mathfrak{J}=\left\{0,1, \mathrm{x}_{\mathrm{a} / 2}, \mathrm{y}_{\mathrm{a} / 4}, \mathrm{x}_{\mathrm{a} / 2} \cup\right.$ $\left.\mathrm{y}_{\mathrm{a} / 4}\right\}$. It is clear that $(\mathrm{X}, \mathfrak{J})$ is semi-discrete and so by Theorem 4.2, it is DH. On the other hand, since $\mathfrak{J}_{\mathrm{a}}=$ $\{\varnothing, X\}$, then $\left(X, \widetilde{J}_{\mathrm{a}}\right)$ is not DH. 


\section{REFERENCES}

[1] L. A. Zadeh, "Fuzzy Sets," Inform and control, vol. 8, pp. 338-353, 1965.

[2] C. L. Chang, "Fuzzy Topological Spaces," Journal of Mathematical Analysis and Applications, vol. 24, pp. 182-190, 1968.

[3] S. Al Ghour, "Homogeneity in fuzzy spaces and their induced spaces," Questions and Answers in General Topology, vol. 21, pp. 185-195, 2003.

[4] S. Al Ghour, "SLH fuzzy spaces," African Diaspora Journal of Mathematics, vol. 2, pp. 61-67, 2004.

[5] S. Al Ghour, A. Fora, "Minimality and Homogeneity in Fuzzy Spaces," Journal of Fuzzy Mathematics, vol. 12, pp. 725--737, 2004.

[6] S. Al Ghour, "Local homogeneity in fuzzy topological spaces," International Journal of Mathematics and Mathematical Sciences, Art. ID 81497, 14 pp, 2006.

[7] S. Al Ghour, "Some Generalizations of Minimal Fuzzy Open Sets," Acta Mathematica Universitatis Comenianae, vol. 75, pp. 107-117, 2006.

[8] S. Al Ghour, K. Al-Zoubi, "On some ordinary and fuzzy homogeneity types," Acta Mathematica Universitatis Comenianae, vol. 77, pp. 199-208, 2008.

[9] S. Al Ghour, A. Fora, "On CDH fuzzy spaces," Journal of Intelligent \& Fuzzy Systems, vol. 30, pp. 935-941, 2016.

[10] A. Fora, S. Al Ghour, "Homogeneity in Fuzzy Spaces," Questions and Answers in General Topology, vol. 19, pp. 159-164, 2001.

[11] S. Al Ghour, A. Azaizeh, "Fuzzy Homogeneous Bitopological Spaces," International Journal of Electrical and Computer Engineering, vol. 8, pp. 2088-8708, 2018.

[12] R. Bennett, "Countable dense homogeneous spaces," Fundamenta Mathematicae, vol. 74, pp.189-194, 1972.

[13] A.V. Arhangel'skii, J. van Mill, "On the cardinality of countable dense homogeneous spaces," Proceedings of the American Mathematical Society, vol. 141, pp. 4031-4038, 2013.

[14] R. Hernandez-Gutiérrez, "Countable dense homogeneity and the double arrow space," Topology and Its Applications, vol. 160, pp. 1123-1128, 2013.

[15] R. Hernandez-Gutiérrez, M. Hrušak, "Non-meager P-filters are countable dense homogeneous," Colloquium Mathematicum, vol. 130, pp. 281-289, 2013.

[16] R. Hernandez-Gutiérrez, M. Hrušak, J. van Mill, "Countable dense homogeneity and $\lambda$-sets," Fundamenta Mathematicae, vol. 226, pp. 157-172, 2014.

[17] M. Hrusak, J. van Mill, "Nearly countable dense homogeneous spaces," Canadian Journal of Mathematics, vol. 66, pp. 743-758, 2014.

[18] J. van Mill, "On countable dense and n-homogeneity," Canadian Mathematical Bulletin, vol. 56, pp. 860-869, 2013.

[19] J. van Mill, "Countable dense homogeneous rimcompact spaces and local connectivity," Filomat, vol. 29, pp. 179-182, 2015.

[20] D. Repovš, L. Zdomskyy, S. Zhang, "Countable dense homogeneous filters and the Menger covering property," Fundamenta Mathematicae, vol. 224, pp. 233-240, 2014.

[21] B. Fitzpatrick, N. F. Lauer, "Densely homogeneous spaces (I)," Houston Journal of Mathematics, vol. 13, pp. 19-25, 1987.

[22] S. K. Cho, "Some results related to densely homogeneous spaces," Communications of the Korean Mathematical Society, vol. 11, pp. 1061-1066, 1996.

[23] D. L. Fearnley, "A Moore space with a $\sigma$-discrete $\pi$-base which cannot be densely embedded in any Moore space with the Baire property," Proceedings of the American Mathematical Society, vol. 127, pp. 3095-3100, 1999.

[24] B. Fitzpatrick, H. X. Zhou, "Densely homogeneous spaces. (II)," Houston Journal of Mathematics, vol. 14, pp. 57-68, 1988

[25] B. Fitzpatrick, H. X. Zhou, "Some open problems in densely homogeneous spaces," Open problems in topology, North-Holland, Amsterdam, pp. 251-259, 1990.

[26] B. Fitzpatrick, J. White, H. X. Zhou, "Homogeneity and $\sigma$-discrete sets," Topology and Its Applications, vol. 44, pp. 143-147, 1992.

[27] S. V. Medvedev, "Metrizable DH-spaces of the first category," Topology and Its Applications, vol. 179, pp. 171-178, 2015.

[28] W. L. Saltsman, "Components of densely homogeneous spaces," Houston Journal of Mathematics, vol. 18, pp. 417-422, 1992.

[29] C. K. Wong, "Fuzzy points and local properties of fuzzy topology," Journal of Mathematical Analysis and Applications, vol. 46, pp. 316-328, 1974.

[30] R. Srivastava, S.N. Lal, A.K. Srivastava, "Fuzzy Hausdorff topological spaces," Journal of Mathematical Analysis and Applications, vol. 81, pp. 497-506, 1981.

[31] M. H.Ghanim, E. E. Kerre, A. S. Mashhour, "Separation axioms, subspaces and sums in fuzzy topology," Journal of Mathematical Analysis and Its Applications, vol. 102, pp. 189-202, 1984.

[32] A. Fora, "Separation axioms for fuzzy spaces," Fuzzy Sets and Systems, vol. 33, pp. 59-75, 1989

[33] R. Lowen, "A comparison of different compactness notions in fuzzy topological spaces," Journal of Mathematical Analysis and Its Applications, vol. 64, pp. 446-454, 1978.

[34] G. J. Wang, "Theory of L-fuzzy topological space," Shanxi Normal University Press, Xian, (in Chinese), 1988.

[35] A. Fora, "Separation axioms, subspaces and product spaces in fuzzy topology," Arab Gulf Journal for Scientific Research, vol. 8, pp. 1-16, 1990. 